\title{
Editorial
}

\section{The Journal Develops}

In recent years papers on neonatal medicine have formed a large proportion of our publications. This reflects the increasing opportunities for investigation and treatment, and for research. In this country most paediatricians have responsibilities both for the newborn and older children and so it is essential that the Journal should cover both areas comprehensively while encouraging aspects which have a high potential for growth.

Clinicians and research workers need to know about advances in the new genetics, physiology and clinical care of the fetus and newborn, animal research, epidemiology, and neurodevelopment. Important advances in research are often made when workers from different disciplines collaborate. The management and editorial committees of the 'Archives' in agreeing to develop the Journal hope to encourage this interaction.

Four additional editions of the 'Archives' will be published each year dealing exclusively with papers that have a broad fetal and neonatal interest. While some papers in these areas will continue to be published in the standard edition, more technical papers will appear in the new edition. The extra editions will retain the Archives of Disease in Childhood title and the same format, with the papers running consecutively with a single yearly index. The additional words Fetal and Neonatal Edition will appear on the cover.

The decision on whether a particular paper is published in the standard or fetal and neonatal edition will be taken by the editors. Publication in the fetal and neonatal edition will not influence the prestige of that paper as it will still be cited as being published in the Archives of Disease in Childhood.

Both editions of the Journal for a particular month will be sent together and there will be no possibility of obtaining one edition without the other. The number of pages in the standard edition will not decrease and therefore there will be more space to accept general paediatric papers of high quality.

The new editions will be managed by the present editors, associate editors, and editorial board. A small number of additional experts may be invited to join the editiorial board, depending on the type of papers submitted.

We invite authors in the fields of genetics including dysmorphism, fetal physiology and medicine, antenatal assessment including imaging techniques, the newborn including animal research relevant to clinical. practice, perinatal epidemiology, and neurodevelopmental research to submit their papers for the new editions of the 'Archives' which will appear in 1988. 